\title{
Generic Self-Similar Blowup for Equivariant Wave Maps and Yang-Mills Fields in Higher Dimensions
}

\author{
Piotr Bizoń ${ }^{1,2}$, Pawel Biernat ${ }^{3}$ \\ ${ }^{1}$ Institute of Physics, Jagiellonian University, Kraków, Poland. E-mail: bizon@th.if.uj.edu.pl \\ 2 Max Planck Institute for Gravitational Physics (Albert Einstein Institute), Golm, Germany \\ 3 Mathematisches Institut, Universität Bonn, Bonn, Germany. E-mail: pawel.biernat@gmail.com
}

Received: 19 December 2014 / Accepted: 25 February 2015

Published online: 6 June 2015 - () The Author(s) 2015. This article is published with open access at Springerlink.com

Abstract: We consider equivariant wave maps from the $(d+1)$-dimensional Minkowski spacetime into the $d$-sphere for $d \geq 4$. We find a new explicit stable self-similar solution and give numerical evidence that it plays the role of a universal attractor for generic blowup. An analogous result is obtained for the $S O(d)$ symmetric Yang-Mills field for $d \geq 6$.

\section{Introduction}

This paper is concerned with the Cauchy problem for the semilinear radial wave equation in $(d+1)$ dimensions

$$
u_{t t}=u_{r r}+\frac{d-1}{r} u_{r}-\frac{f(u)}{r^{2}},
$$

where $r \geq 0$ is the radial variable and $u=u(t, r)$. For concreteness, we shall focus on two specific and particularly interesting cases:

$$
f(u)= \begin{cases}(d-1) \sin (u) \cos (u), & (\mathrm{WM}) \\ d u(1-u)(2-u), & (\mathrm{YM})\end{cases}
$$

which correspond to equivariant wave maps from the $(d+1)$-dimensional Minkowski spacetime into the $d$-sphere and an $S O(d+2)$ symmetric Yang-Mills field in the $(d+2)+1$ dimensional Minkowski spacetime, respectively (see e.g. [1] for the derivation).

The basic question for Eq. (1) is whether solutions starting from smooth initial data can become singular ("blow up") in finite time and, if so, how does the blowup occur. The key feature, which plays a major role in answering this question, is the scale invariance of Eq. (1) with respect to the scaling transformation $u(t, r) \mapsto u_{L}(t, r)=u(t / L, r / L)$, where $L$ is a positive constant. Under this scaling the conserved energy 


$$
E(u)=\int_{0}^{\infty}\left(\frac{1}{2} u_{t}^{2}+\frac{1}{2} u_{r}^{2}+\frac{F(u)}{r^{2}}\right) r^{d-1} d r,
$$

where $F^{\prime}(u)=f(u)$, transforms as $E\left(u_{L}\right)=L^{d-2} E(u)$, which means that Eq. (1) is energy critical for $d=2$ and supercritical for $d \geq 3$.

The critical case $d=2$ has been intensively studied for the past decades leading to a good understanding of the mechanism of blowup. Most notably, Struwe proved that singularities necessarily have the form of self-shrinking harmonic maps (hence nonexistence of harmonic maps implies global regularity) and the rate of shrinking is faster than self-similar [2]. For the nonlinearities (2) the generic rates of shrinking were derived formally in $[3,4]$ and proved rigorously in [5].

The understanding of the supercritical case $d \geq 3$ is much less satisfactory. All known examples of blowup involve self-similar solutions [1,6-9]; however, it is not known if singularities must be self-similar. For $d=3$ and the nonlinearities (2), the self-similar blowup was proved (under a certain spectral condition ${ }^{1}$ ) to be stable $[10,11]$ and demonstrated numerically to be generic $[12,13]$. In higher dimensions, as far as we know, nothing was known in even dimensions, while for odd $d \geq 5$ some self-similar solutions have been constructed $[1,8]$ but their stability and role in dynamics have not been studied.

In this paper we present new explicit self-similar solutions of Eq. (1) in all dimensions $d \geq 4$ (Sect. 2), establish their linear stability (Sect. 3), and finally give numerical evidence that they play the role of universal attractors in the generic blowup (Sect. 4).

\section{Self-Similar Solutions}

By definition, self-similar solutions are invariant under the scaling, that is $u(t / L, r / L)=$ $u(t, r)$, hence they have the form

$$
u(t, r)=\phi(y), \quad y=\frac{r}{T-t},
$$

where a positive constant $T$, clearly allowed by the time translation symmetry, is introduced for later convenience. Inserting this ansatz into Eq. (1) one obtains the ordinary differential equation

$$
\left(1-y^{2}\right) \phi^{\prime \prime}+\left(\frac{d-1}{y}-2 y\right) \phi^{\prime}-\frac{f(\phi)}{y^{2}}=0 .
$$

We are interested in solutions that are smooth on the closed interval $0 \leq y \leq 1$, which corresponds to the interior of the past light cone of the point $(t=T, r=0)$. For such solutions

$$
\left.\frac{\partial^{n}}{\partial r^{n}} \phi\left(\frac{r}{T-t}\right)\right|_{r=0}=(T-t)^{-n} \frac{d^{n} \phi}{d y^{n}}(0),
$$

hence the $n$-th derivative at the origin ( such that $\frac{d^{n} \phi}{d y^{n}}(0) \neq 0$ ) diverges as $t \nearrow T$. Thus, each self-similar solution $\phi(y) \in C^{\infty}[0,1]$ is an example of a singularity developing in finite time from smooth initial data.

\footnotetext{
1 Recently, Costin et al. [14] have verified the spectral assumption in question, thereby completing the nonlinear stability proof of Donninger.
} 
We found the following explicit solutions of Eq. (5)

$$
\phi_{0}(y)=\left\{\begin{array}{l}
2 \arctan \left(\frac{y}{\sqrt{d-2}}\right), \\
\frac{a y^{2}}{y^{2}+b},
\end{array}\right.
$$

where

$$
a=\frac{2(d+4-\sqrt{3 d(d-2)})}{3 d-2 \sqrt{3 d(d-2)}}, \quad b=\frac{1}{3} \frac{\sqrt{3 d(d-2)}(8-d)}{3 d-2 \sqrt{3 d(d-2)}} .
$$

For $d=3$ these solutions have been known [7,9], however for $d \geq 4$ they appear to be new. In particular, the only smooth self-similar solutions obtained previously, for $d=5$ (WM) and $d \in\{5,7\}(\mathrm{YM})$, by variational [1] and shooting [8] methods are different from $\phi_{0}$. For both these methods it was essential that $\phi(1)=\phi_{*}$, where $\phi_{*}$ is the convexity radius of the target manifold, equal to $\pi / 2$ for (WM) and 1 for (YM). In contrast, for our solutions $\phi_{0}(1)<\phi_{*}$ when $d \geq 4$.

Remark 1. A complete classification of smooth solutions of Eq. (5) for $d \geq 4$ is not an easy problem; the main difficulty being due to an involved smoothness condition at $y=1$ (see section 2.2 in [1] for the analysis of this condition in odd dimensions). Fortunately, this hard ODE problem need not concern us here because, as will be shown in Sect. 4, smooth solutions other than $\phi_{0}$ do not seem to participate in the generic blowup, presumably due to their instabilities.

Remark 2. Clearly, solutions similar to $\phi_{0}$ exist for a variety of nonlinearities other than (2) (albeit not in an explicit form, of course). In particular, by modifying (2) for $\phi>\phi_{0}(1)$ one can construct analogous smooth solutions for convex targets using the shooting method of [1].

Remark 3. It is instructive to compare the problem of blowup for Eq. (1) with the analogous problem for the heat flow

$$
u_{t}=u_{r r}+\frac{d-1}{r} u_{r}-\frac{f(u)}{r^{2}}
$$

with the same nonlinearities (2). This equation has self-similar blowup solutions for $3 \leq d \leq 6$ (WM) [18] and $3 \leq d \leq 7$ (YM) [19] but no such solutions exist in higher dimensions [20]. In accordance with that, it was found that the blowup for Eq. (8) changes character from self-similar [21] to non-self-similar [22] in sufficiently high dimensions.

\section{Linear Stability Analysis}

As the first step towards understanding the role of the self-similar solution $\phi_{0}\left(\frac{r}{T-t}\right)$ in dynamics we need to analyze its linear stability. To this end it is convenient to define a new time coordinate $s=-\ln (T-t)$ and rewrite Eq. (1) in terms of $U(s, y)=u(t, r)$

$$
U_{s s}+U_{s}+2 y U_{s y}=\left(1-y^{2}\right) U_{y y}+\left(\frac{d-1}{y}-2 y\right) U_{y}-\frac{f(U)}{y^{2}} .
$$


In these variables the problem of finite time blowup is converted into the problem of asymptotic convergence for $s \rightarrow \infty$ towards the stationary solution $\phi_{0}(y)$. Following the standard procedure we seek solutions of Eq. (9) in the form $U(s, y)=\phi_{0}(y)+e^{\lambda s} v(y)$. Dropping nonlinear terms we get the quadratic eigenvalue equation

$$
\left(1-y^{2}\right) v^{\prime \prime}+\left(\frac{d-1}{y}-2(\lambda+1) y\right) v^{\prime}-\lambda(\lambda+1) v-V(y) v=0,
$$

where

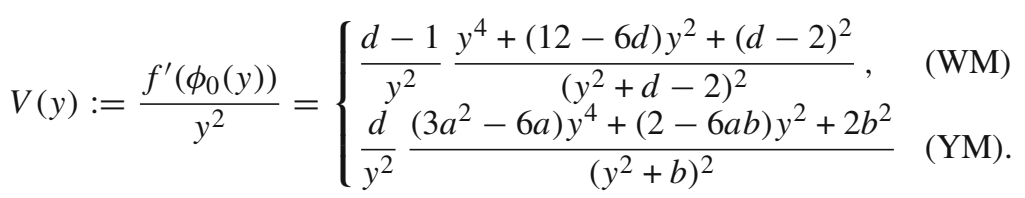

We consider the eigenvalue problem (10) on the interval $0 \leq y \leq 1$. By assumption, the solution $U(s, y)$ is smooth for $s<\infty$, hence we we demand that $v \in \mathcal{C}^{\infty}[0,1]$. This condition leads to the quantization of the eigenvalues.

In order to determine the analyticity properties of solutions of Eq. (10) it is convenient to introduce new variables $x$ and $w(x)$ defined by

$$
x=\frac{c y^{2}}{y^{2}+c-1}, \quad v(y)=x^{\mu}(c-x)^{\frac{\lambda}{2}} w(x),
$$

where $\mu=1 / 2, c=d-1$ for (WM) and $\mu=1, c=b+1$ for (YM). This change of variables brings Eq. (10) into the canonical form of the Heun equation [23]

$$
w^{\prime \prime}+\left(\frac{\gamma}{x}+\frac{\delta}{x-1}+\frac{\epsilon}{x-c}\right) w^{\prime}+\frac{\alpha \beta x-q}{x(x-1)(x-c)} w=0,
$$

where in the case of (WM)

$$
\begin{aligned}
& \gamma=1+d / 2, \quad \delta=(3-d) / 2+\lambda, \quad \epsilon=d / 2, \quad \alpha=(\lambda+5) / 2, \\
& \beta=(\lambda-1) / 2, \quad q=(\lambda-1)(d \lambda+5 d-2 \lambda-6) / 4,
\end{aligned}
$$

and in the case of (YM)

$$
\begin{aligned}
& \gamma=2+d / 2, \quad \delta=(3-d) / 2+\lambda, \quad \epsilon=1 / 2, \quad \alpha=3+\lambda-\beta, \\
& \beta=\frac{1}{2 b+2}\left(b \lambda+\left(3 a^{2} b d+3 a^{2} d+b^{2}+2 b+1\right)^{\frac{1}{2}}+3 b+\lambda+3\right), \\
& q=b \lambda^{2} / 4-3 a d / 2+5 b \lambda / 4+d \lambda / 4+3 b / 2+d / 2+\lambda+2 .
\end{aligned}
$$

Equation (12) has four regular singularities at $0,1, c, \infty$. The characteristic exponents at 0 and 1 are $\{0,1-\gamma\}$ and $\{0,1-\delta\}$, respectively. The regular solutions at $x=0$ and $x=1$ are given by the local Heun functions (using the Maple notation)

$$
\begin{aligned}
& w_{0}(x)=\operatorname{HeunG}(c, q, \alpha, \beta, \gamma, \delta ; x), \\
& w_{1}(x)=\operatorname{HeunG}(1-c,-q+\alpha \beta, \alpha, \beta, \delta, \gamma ; 1-x) .
\end{aligned}
$$

The eigenvalues are given by zeros of the Wronskian

$$
W\left[w_{0}, w_{1}\right](\lambda):=w_{0}^{\prime}(x) w_{1}(x)-w_{1}^{\prime}(x) w_{0}(x) .
$$


Table 1. The first few eigenvalues $\lambda_{n}(d)$ for the (WM) case

\begin{tabular}{llllll}
\hline & $n=0$ & $n=1$ & $n=2$ & $n=3$ & $n=4$ \\
\hline$d=3$ & 1 & -0.542466 & -2.000000 & -3.398381 & -4.765079 \\
$d=4$ & 1 & -0.563612 & -2.109131 & -3.603718 & -5.061116 \\
$d=5$ & 1 & -0.572315 & -2.163011 & -3.711951 & -5.216059 \\
$d=6$ & 1 & -0.577089 & -2.195673 & -3.780281 & -5.306294 \\
$d=7$ & 1 & -0.580109 & -2.217711 & -3.827722 & -5.354120 \\
$d=8$ & 1 & -0.582193 & -2.233621 & -3.862716 & -5.367078 \\
\hline
\end{tabular}

Table 2. The first few eigenvalues $\lambda_{n}(d)$ for the (YM) case

\begin{tabular}{llllll}
\hline & $n=0$ & $n=1$ & $n=2$ & $n=3$ & $n=4$ \\
\hline$d=3$ & 1 & -0.588904 & -2.181597 & -3.570756 & -5.043294 \\
$d=4$ & 1 & -0.623081 & -2.255420 & -3.765173 & -5.326428 \\
$d=5$ & 1 & -0.636360 & -2.295861 & -3.867878 & -5.479403 \\
$d=6$ & 1 & -0.643402 & -2.321548 & -3.932725 & -5.577793 \\
$d=7$ & 1 & -0.647756 & -2.339350 & -3.977728 & -5.647093 \\
$d=8$ & 1 & -0.650712 & -2.352428 & -4.010905 & -5.698800 \\
\hline
\end{tabular}

Unfortunately, the connection problem for the Heun equation is unresolved and therefore (in contrast to the hypergeometric equation) no explicit formula for the Wronskian is available. Fortunately, the Heun functions are built into Maple and the zeros of the Wronskian can be computed numerically with great precision. ${ }^{2}$ The results of this computation are displayed in Table 1 (WM) and Table 2 (YM).

We remark that the eigenvalue $\lambda_{0}=1$ is due to the freedom of changing the blowup time $T$ and does not correspond to any instability. The fact that the corresponding eigenfunction $v_{0}(y)=y \phi_{0}^{\prime}(y)$ has no zeros can be used to exclude rigorously eigenvalues with $\Re(\lambda)>d-2($ see $[15,16]$ for the proof in $d=3)$, however it seems hard to prove the nonexistence of eigenvalues with $0<\Re(\lambda)<d-2$. Nonetheless, we feel confident that if such an eigenvalue existed, we would have found it numerically. Consequently, we assume that all the eigenvalues but $\lambda_{0}$ have negative real part. ${ }^{3}$ From this assumption it follows that the solution $\phi_{0}$ is linearly asymptotically stable [16].

\section{Dynamics of Blowup}

In the lowest supercritical dimension $d=3$ Donninger proved that the linear asymptotic stability of $\phi_{0}$ implies its nonlinear asymptotic stability [10,11]. It seems feasible that this result can be generalized to higher dimensions $d \geq 4$ but we are not qualified to pursue this issue. We conjecture that the solution $\phi_{0}$ is not only nonlinearly stable but, in fact, it is a universal attractor for generic blowup. The numerical evidence for this conjecture in the case $d=3$ was given in $[12,13]$; here we give an analogous evidence for $d \geq 4$.

Consider a solution $u(t, r)$ of Eq. (1) that starts from smooth initial data at $t=0$ and becomes singular at $(r=0, t=T)$ for some $T>0$ (recall that singularities at $r>0$ are impossible). We want to show that generically $u(t, r)$ converges to the self-similar solution $\phi_{0}\left(\frac{r}{T-t}\right)$ as $t \nearrow T$ and the rate and profile of convergence are determined by the least damped mode $e^{\lambda_{1} s} v_{1}(y)$, that is

\footnotetext{
2 An alternative way is to use the method of continued fractions [15]. We have verified that these two methods give identical results.

3 The methods from [14] and, in particular, very recent [17] would seem relevant in proving this assumption.
} 


$$
u(t, r)-\phi_{0}\left(\frac{r}{T-t}\right) \sim C(T-t)^{-\lambda_{1}} v_{1}\left(\frac{r}{T-t}\right),
$$

where the coefficient $C$ and blowup time $T$ depend on the initial data.

To verify (16), we solved Eq. (1) numerically for several large, 'randomly' chosen, initial data leading to blowup. In order to keep track of the structure of the singularity developing on progressively smaller scales, it is necessary to use an adaptive method which refines the spatio-temporal grid near the singularity. Our numerical method is based on the moving mesh method (known as MMPDE6, see [24]) combined with the Sundman transformation, as described in [25], with some minor modifications and improvements specific to the problem at hand. This method is reasonably efficient in computations of self-similar singularities.

After obtaining a numerical solution $u(r, t)$ we estimate the blow-up time $T$ and pass to the similarity variables $s=-\log (T-t), y=r /(T-t)$ and $U(y, s)=u(r, t)$ in order to compare analytical and numerical results. The results are illustrated in Figs. 1 and 2.

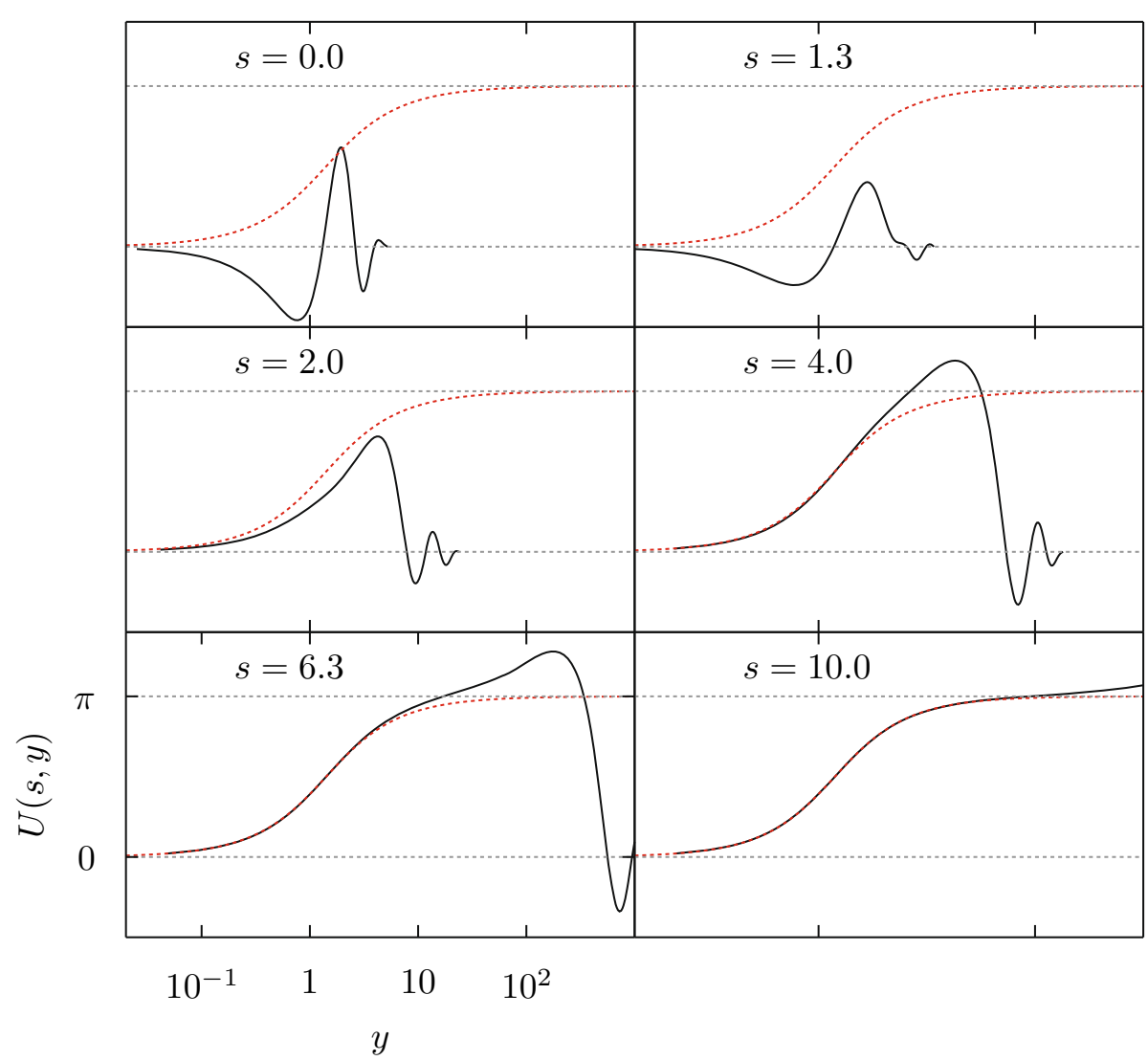

Fig. 1. Snaphots from the evolution of sample initial data leading to blowup for the (WM) case in $d=4$. The solution $u(t, r)=U(s, y)$ converges to the self-similar solution $\phi_{0}(y)=2 \arctan (y / \sqrt{2})$ (dashed red curve) (color figure online) 

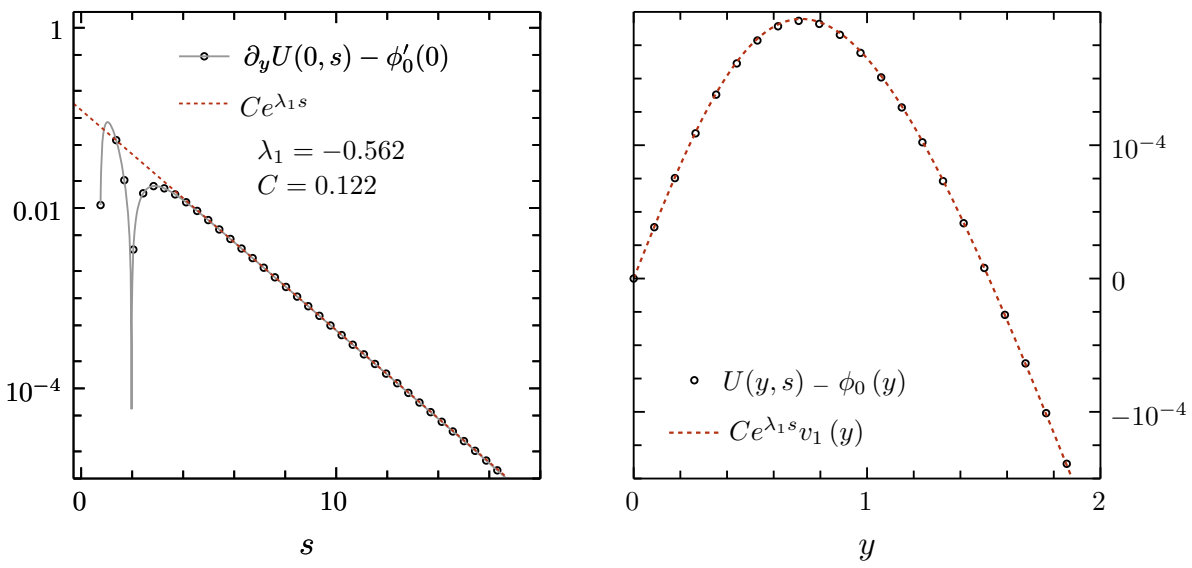

Fig. 2. Numerical verification of the formula (16) for the solution depicted in Fig. 1. Left panel We plot $(T-t) \partial_{r} u(t, 0)-\sqrt{2}$ in $\log -\log$ scale and fit $C(T-t)^{-\lambda_{1}}$. The value of $\lambda_{1}$ obtained from the fit agrees within one percent with the value given in Table 1. Right panel For the parameters $C$ and $\lambda_{1}$ determined above, the left and right hand sides of (16) are shown to agree at time $s=4$

Acknowledgements. P. Bizoń is supported by the Polish National Science Centre Grant no. DEC-2012/06/A/ ST2/00397. P. Biernat is supported by the Alexander von Humboldt Foundation in the framework of Roland Donninger's Sofja Kovalevskaja Award endowed by the German Federal Ministry of Education and Research. P. Bizoń acknowledges the hospitality of the Banff International Research Station (Canada), where this work was initiated.

Open Access This article is distributed under the terms of the Creative Commons Attribution 4.0 International License (http://creativecommons.org/licenses/by/4.0/), which permits unrestricted use, distribution, and reproduction in any medium, provided you give appropriate credit to the original author(s) and the source, provide a link to the Creative Commons license, and indicate if changes were made.

\section{References}

1. Cazenave, T., Shatah, J., Tahvildar-Zadeh, A.S.: Harmonic maps of the hyperbolic space and the development of singularities in wave maps and Yang-Mills fields. Ann. Inst. Henri Poincaré 68, 315 (1998)

2. Struwe, M.: Equivariant wave maps in two space dimensions. Commun. Pure Appl. Math. 56(7), 815 (2003)

3. Bizoń, P., Ovchinnikov, Y.N., Sigal, I.M.: Collapse of an instanton. Nonlinearity 17, 1179 (2004)

4. Ovchinnikov, Y.N., Sigal, I.M.: On collapse of wave maps. Phys. D 240, 1311 (2011)

5. Raphaël, P., Rodnianski, I.: Stable blow up dynamics for the critical corotational wave maps and equivariant Yang Mills problems. Publ. Math. Inst. Hautes Etudes Sci. 115, 1 (2012)

6. Shatah, J.: Weak solutions and development of singularities in the $S U(2) \sigma$-model. Commun. Pure Appl. Math. 41, 459 (1988)

7. Turok, N., Spergel, D.: Global texture and the microwave background. Phys. Rev. Lett. 64, 2736 (1990)

8. Bizoń, P.: Equivariant self-similar wave maps from Minkowski spacetime into 3-sphere. Commun. Math. Phys. 215, 45 (2000)

9. Bizoń, P.: Formation of singularities in Yang-Mills equations. Acta Phys. Polon. B 33, 1893 (2002)

10. Donninger, R.: On stable self-similar blowup for equivariant wave maps. Commun. Pure Appl. Math. 64, 1095 (2011)

11. Donninger, R.: Stable self-similar blowup in energy supercritical Yang-Mills theory. Math Z. 278(34), 1005-1032 (2014)

12. Bizoń, P., Chmaj, T., Tabor, Z.: Dispersion and collapse of wave maps. Nonlinearity 13, 1411 (2000)

13. Bizoń, P., Tabor, Z.: On blowup of Yang-Mills fields. Phys. Rev. D 64, 121701 (2001)

14. Costin, O., Donninger, R., Xia, X.: A proof for the mode stability of a self- similar wave map. arXiv: 1411.2947

15. Bizoń, P.: An unusual eigenvalue problem. Acta. Phys. Polon. B 36, 5 (2005) 
16. Donninger, R., Schörkhuber, B., Aichelburg, P.C.: On stable self-similar blow up for equivariant wave maps: the linearized problem. Ann. Henri Poincaré 13, 103 (2012)

17. Costin, O., Donninger, R., Glogić, I., Huang, M.: On the stability of self-similar solutions to nonlinear wave equations. arXiv: 1502.06280

18. Fan, H.: Existence of the self-similar solutions in the heat flow of harmonic maps. Sci. China Ser. A 42, 113 (1999)

19. Weinkove, B.: Singularity formation in the Yang-Mills flow. Calc. Var. Part. Differ. Equ. 19, 211 (2004)

20. Bizoń, P., Wasserman, A.: Non-existence of shrinkers for the harmonic map flow in higher dimensions. Int. Math. Res. Not. (2014). doi:10.1093/imrn/rnu176

21. Biernat, P., Bizon, P.: Shrinkers, expanders, and the unique continuation beyond generic blowup in the heat flow for harmonic maps between spheres. Nonlinearity 24, 2211 (2011)

22. Biernat, P.: Non-self-similar blow-up in the heat flow for harmonic maps in higher dimensions. Nonlinearity 28, 167-185 (2015)

23. NIST Digital Library of Mathematical Functions. http://dlmf.nist.gov/31.2. Accessed 29 Aug 2014

24. Huang, W., Ren, Y., Russell, R.D.: Moving mesh partial differential equations (MMPDES) based on the equidistribution principle. SIAM J. Numer. Anal. 31, 709 (1994)

25. Budd, C.J., Williams, J.F.: How to adaptively resolve evolutionary singularities in differential equations with symmetry. J. Eng. Math. 66, 217-236 (2010)

Communicated by W. Schlag 